\title{
Ambulation and physical function after eccentric resistance training in adults with incomplete spinal cord injury: A feasibility study
}

\section{Whitley J. Stone ${ }^{1}$, Sandra L. Stevens ${ }^{2}$, Dana K. Fuller ${ }^{3}$, Jennifer L. Caputo ${ }^{2}$}

\author{
${ }^{1}$ Nutrition and Kinesiology, University of Central Missouri, Warrensburg, Missouri, USA, ${ }^{2} \mathrm{Health}$ and Human \\ Performance, Middle Tennessee State University, Murfreesboro, Tennessee, USA, ${ }^{3}$ Psychology, Middle Tennessee \\ State University, Murfreesboro, Tennessee, USA
}

Background: Strengthening the lower extremities has shown to positively influence walking mechanics in those with neurological deficiencies. Eccentric resistance training (ERT) is a potent stimulus for the development of muscular strength with low metabolic demand. Thereby, ERT may benefit those with incomplete spinal cord injuries (iSCl) seeking to improve ambulatory capacity.

Design: This study was aimed to determine the effect of ERT on walking speed, mobility, independence, and at home function following iSCl.

Methods: Individuals with longstanding iSCI trained twice a week for 12 weeks on an eccentrically biased recumbent stepper.

Outcome measures: Walking speed (10 meter walk test; 10MWT), mobility (timed up and go), independence (Walking Index for Spinal Cord Injury; WISCI), and at home function (Spinal Cord Independence Measure; SCIM) were assessed at baseline, after 6 weeks, and after 12 weeks of ERT.

Results: There were improvements in walking mobility $(158.36+165.84$ seconds to $56.31+42.42$ seconds, $P=.034, d=0.62)$, speed $(0.34+0.42 \mathrm{~m} / \mathrm{s}$ to $0.43+0.50 \mathrm{~m} / \mathrm{s}, P=.005, d=.23)$, and independence $(8+7$ to $13+7, P=.004, d=.73)$ after 12 weeks of ERT. At home function remained unchanged $(22+10$ to $24+10, P=.10, d=.12$ ).

Conclusions: Improving lower extremity strength translated to walking performance and independence in those with iSCI. Additionally, ERT may diminish therapist burden in programs designed to improve ambulatory capacity or strength in those with iSCl.

Keywords: Paraplegia, Resistance training, Exercise, Lower extremity, Neurology

\section{Introduction}

Motor impairment following an incomplete spinal cord injury (iSCI) often leads to compromised gait, decreased muscular strength, and altered metabolic function. ${ }^{1}$ The resulting decreases in mobility and independence lead to increased risk of hypokinetic diseases. ${ }^{2}$ To help combat the deleterious effects of a sedentary lifestyle, as in those without disability, it is recommended that individuals with iSCI participate in cardiovascular activities

Correspondence to: Whitley J. Stone, Ph.D. Nutrition and Kinesiology, University of Central Missouri, Warrensburg, MO, USA. E-mail: whstone@ ucmo.edu

This article has been republished with minor changes. These changes do not impact the academic content of the article. most days of the week supplemented with muscular strengthening exercises. ${ }^{3-5}$

In addition to improving health, active lower body resistance training (RT) may also improve ambulatory capacity after iSCI. Unfortunately, the attenuated muscular force capacity following more severe iSCI may be insufficient to stimulate muscular adaptations necessary for RT or ambulation. Often, those with iSCI perform RT through neuromuscular electrical stimulation (NMES). Investigators have noted improvements in strength and body composition after NMES RT following motor complete SCI. ${ }^{6-8}$ However, chronic use of NMES may lead to reliance on the external stimuli rather than in vivo neuromuscular force generation. Alternately, lower body RT 
may be paired with gait training in order to improve ambulatory capacity, simultaneously training gait mechanics and limb strength. ${ }^{9}$ However, 16 weeks of RT improved walking speed more than gait training in high functioning (AIS D) individuals with longstanding iSCI. ${ }^{10}$

Eccentric resistance training (ERT) may be an optimal training regimen for those with iSCI seeking increased muscular strength without external NMES or therapist assisted training. Compared to concentric contractions, eccentric muscle actions require fewer neurons to fire to generate force, metabolic demand is lower for a given workload, and maximal force production is higher. ${ }^{11}$ The Eccentron (BTETech, Hanover, MD, USA; see Figure 1), a motor driven eccentric stepper; is one modality that may be ideal for those with iSCI. Originally designed to train older adults, athletes, or those who have had cardiopulmonary complications, the Eccentron delivers a controlled and measureable negative muscular overload. The Eccentron has been safely implemented in able-bodied ${ }^{12}$ and special populations such as iSCI. ${ }^{13}$ Training on the Eccentron allows an individual with iSCI to bilaterally work the limbs and visually track the accuracy of force production during repetitions. The Eccentron may assist in concurrently improving lower extremity muscle strength and reciprocal limb activation, both of which may improve ambulatory function.

Although muscular strength is correlated with walking capacity, little is known about the efficacy of ERT on walking speed, mobility, assistance needed during walking tasks, at home daily physical function, and daily step physical activity (DSPA). Further, the relationships between laboratory measurements of these variables and free-living DSPA are unknown. Therefore, the purpose of this study was to evaluate ambulatory measures, physical function, and DSPA before, after 6-weeks, and after 12-weeks of ERT on the Eccentron. It was hypothesized that time on the timed up and go (TUG) and 10 meter walk test (10MWT) would decrease and scores on the Spinal Cord Independence Measure (SCIM) and Walking Independence for Spinal Cord Injury (WISCI) tests would increase. A secondary purpose was to evaluate the relationship between laboratory assessments and DSPA. It was hypothesized that the TUG, 10MWT, WISCI, and SCIM would be predictors of DSPA.

\section{Materials and Methods}

\section{Participants}

The convenience sample included 7 males and 4 females (6 cervical, 4 thoracic, 1 lumbar iSCI) recruited via word of mouth and email. Average age of participants was 39.0 years ( \pm 15.9 years) with an average of 9.5 years
( \pm 4.7) post-iSCI. Additional participant characteristics are provided in Table 1. Volunteers were included pending injury categorized as sensorimotor iSCI (Asia Impairment Scale B-D) for greater than 1 year and able to complete testing protocols (ambulatory and strength tests). Musculoskeletal injury within the past year or currently participating in structured lower body RT were exclusion criteria. The study was approved by the university Institutional Review Board and participants provided informed consent.

\section{Instrumentation}

\section{Anthropometric measure}

Assessments were performed by the same, trained technician. Body mass was measured to the nearest 0.01 kilogram on a standing scale [Health O' Meter $753 \mathrm{KL}$, IL, USA] or wheelchair scale [Seca 674, Hamburg, Germany].

\section{Pain}

Upon arriving to the laboratory each day, participants drew a vertical line on a 10 -centimeter visual analog scale (VAS) to represent lower extremity pain intensity or soreness associated with the training protocol. ${ }^{10}$ Scores ranged from 0 "no pain" to 10 "pain as bad as it can be." The VAS (or numeric rating scale) is valid and reliable in those with chronic pain after iSCI. ${ }^{14}$ Pain was assessed on the first two laboratory visits to establish an average baseline. Scores beyond 9 centimeters or deviations from baseline $>3$ centimeters resulted in delay of any increase in training intensity until the next training session or the participant was given the option to postpone training to another day.

\section{Functional independence}

The ability of participants to complete daily tasks associated with independent living was assessed with the validated SCIM. ${ }^{15-16}$ Highly correlated with the Berg Balance Scale and the Functional Independence Measure in this population, ${ }^{15-16}$ the SCIM allows an individual to earn up to 20 points on self-care, 40 points for respiration and sphincter management, and 40 points for mobility (total score $\leq 100$ ). Participants completed the SCIM after visit one, at mid-assessment, and post-ERT.

\section{Resting tone, eccentric testing, and training}

Eccentron training sessions lasted an average of 15 minutes. This motor driven eccentric ergometer drives the pedals towards the seated individual who eccentrically resists the movement. The Eccentron targets the gluteal, hamstring, and quadriceps muscles. Positioning for testing and training was adjusted so that when the left foot was on the pedal and the leg 


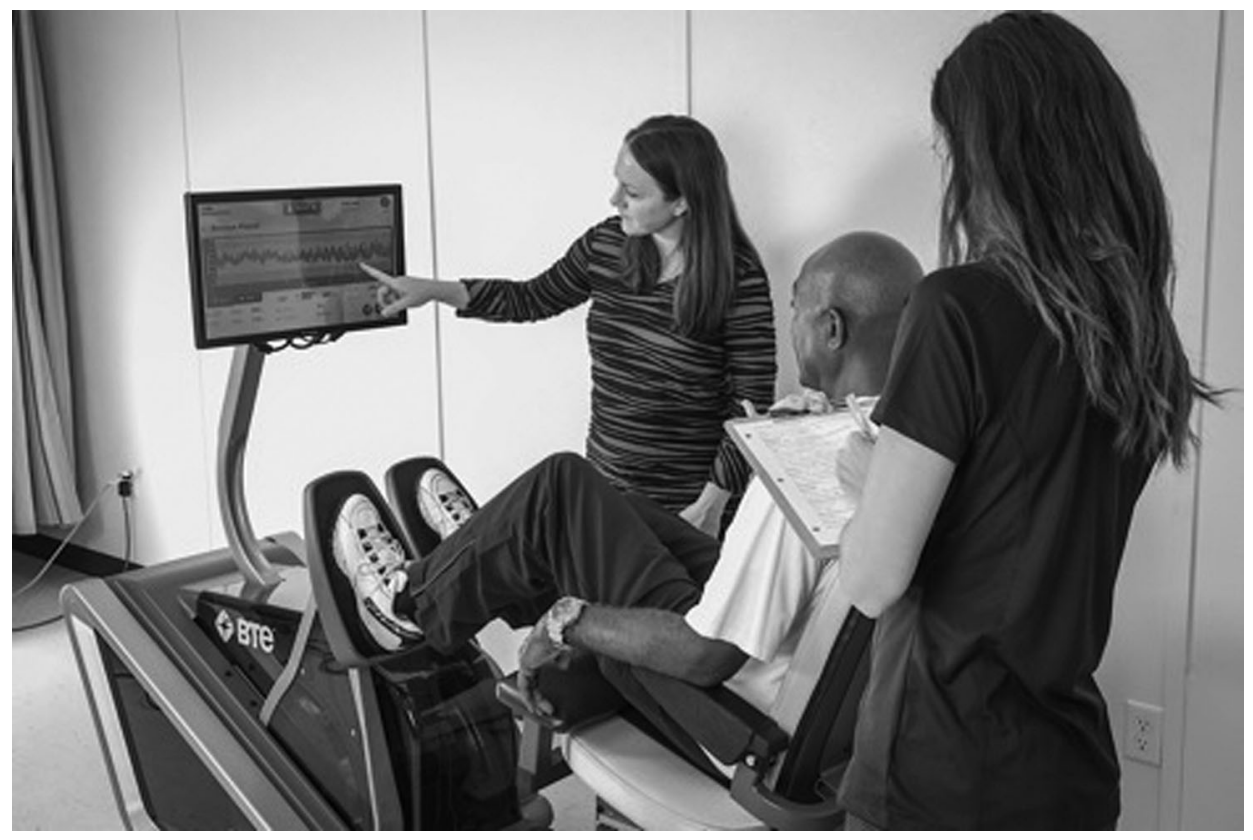

Figure 1 The eccentrically biased training modality. As the pedals isokinetically moved, participants resisted to elicit an eccentric muscle action of the hamstrings and gluteal muscles.

fully extended, the knee angle was $45^{\circ}-55^{\circ}$. While in this position, the contralateral knee was flexed at approximately $90^{\circ}$. After familiarization, but prior to three repetition maximum (3RM) testing, the participant was asked to allow the pedals to passively move for 1 minute. This assessment determined the average force exerted through underlying muscular tone when the legs were inertly moving throughout the range of motion. The participant eccentrically warmed up prior to testing.

Bilateral eccentric strength was determined using a $3 \mathrm{RM}$ test on the Eccentron at 12 repetitions per minute

Table 1 Individual participant characteristics $(N=11)$.

\begin{tabular}{|c|c|c|c|c|c|c|}
\hline Sex & ne po & (years) & $\begin{array}{c}\text { Walking } \\
\text { speed }(\mathrm{m} / \mathrm{s})\end{array}$ & WISCI & $\begin{array}{l}\text { AIS } \\
\text { Score }\end{array}$ & $\begin{array}{l}\text { Primary mode } \\
\text { of locomotion }\end{array}$ \\
\hline $\mathrm{F}$ & 69 & 10.3 & 0.60 & 9 & C & Rolling walker \\
\hline $\mathrm{M}$ & 44 & 8.3 & 0.21 & 6 & C & M. wheel chair \\
\hline $\mathrm{M}$ & 60 & 5.2 & 0.84 & 19 & D & Forearm crutch \\
\hline M & 44 & 8.3 & 0.09 & 6 & $B$ & M. wheel chair \\
\hline$M$ & 26 & 4.9 & 0.03 & 2 & C & M. wheel chair \\
\hline $\mathrm{M}$ & 46 & 9.1 & 0.19 & 9 & C & M. wheel chair \\
\hline $\mathrm{F}$ & 26 & 4.9 & 0.40 & 10 & $\mathrm{D}$ & Cane + AFO \\
\hline M & 36 & 8.8 & 0.02 & 2 & B & M. wheel chair \\
\hline $\mathrm{F}$ & 24 & 8.9 & 0.01 & 2 & B & P. wheel chair \\
\hline $\mathrm{F}$ & 37 & 20.5 & 1.30 & 20 & D & Ambulation \\
\hline M & 21 & 15.0 & 0.02 & 2 & B & M. wheel chair \\
\hline
\end{tabular}

Note. Age in years. Walking speed = baseline 10 meter walk test. WISCI, Walking Index for Spinal Cord Injury; AIS, ASIA Impairment Scale. Primary modes of locomotion included wheeled (rolling) walkers, manual wheel chairs (M. wheel chair), powered wheel chairs (P. wheel chair), forearm crutches, canes with ankle foot orthotics (AFO), and ambulation (with no assistive devices). (rpm). Taking the better of 2 trials on day two, the peak force of the more impaired leg during the 3RM was converted to a $1 \mathrm{RM}$ and used for the initial exercise prescription. ${ }^{17}$ This $3 \mathrm{RM}$ procedure was repeated at the beginning of week three to obtain workload two, week six to establish workload three (midpoint assessment), and after completing the ERT (see Table 2).

\section{Mobility}

The TUG is an ambulatory test designed to assess walking performance and mobility. The TUG has excellent inter/interrater reliability and construct validity in

Table 2 Eccentric resistance training program.

\begin{tabular}{|c|c|c|c|c|}
\hline Week(s) & Training load \% & Sets & Reps & Rest (min) \\
\hline $1-2$ & \multicolumn{4}{|c|}{$\begin{array}{l}\text { Familiarization } \\
\text { **Week } 2 \text { Day 2: 3RM Assessment for workload } 2\end{array}$} \\
\hline $3-5$ & $\begin{array}{l}\text { Day 1: } \geq 85 \\
\text { Day 2: } 67-80\end{array}$ & $\begin{array}{l}2-6 \\
3-6\end{array}$ & $\begin{array}{l}\leq 6 \\
6-10\end{array}$ & $\begin{array}{l}3.0 \\
1.5\end{array}$ \\
\hline 6 & \multicolumn{4}{|c|}{$\begin{array}{l}{ }^{*} \text { Week } 6 \text { Day 2: 3RM Assessment for workload } 3 \text { \& } \\
\text { mid-test data }\end{array}$} \\
\hline $7-9$ & $\begin{array}{l}\text { Day } 1: \geq 85 \\
\text { Day 2: } 67-75\end{array}$ & $\begin{array}{l}2-6 \\
3-6\end{array}$ & $\begin{array}{l}\leq 6 \\
6-10\end{array}$ & $\begin{array}{l}3.0 \\
1.5\end{array}$ \\
\hline $10-12$ & $\begin{array}{l}\text { Day } 1: \geq 85 \\
\text { Day } 2: 70-84\end{array}$ & $\begin{array}{l}5-6 \\
5-6\end{array}$ & $\begin{array}{l}\leq 6 \\
10-12\end{array}$ & $\begin{array}{l}3.0 \\
1.5\end{array}$ \\
\hline
\end{tabular}

Note. Reps = repetitions; $\min =$ minutes; Day $1=$ muscular strength; Day 2 = muscular hypertrophy. Resistance = percent of the peak mean force of the weaker leg during the respective 3RM. Rest $=$ time between sets. Weeks 1 and 2 were a familiarization period where participants chose training duration and intensity, but were advised to work around $2 \times 8$ at $40 \%$ of Workload 1 at $12 \mathrm{rpm}$. 
populations with iSCI (highly correlated with WISCI II and 6 minute walk test). ${ }^{18}$ As quickly and safely as possible, the participant stood from a chair, walked 3 meters, turned, and returned to a seated position. The participant used the least amount of assistance (physical or device) needed to safely complete the task. Any assistive device(s) used during pre-testing was also used at midand post-testing. Time to complete the TUG was recorded in seconds. Participant completed up to 3 trials and the fastest attempt was recorded for data analyses.

\section{Walking speed}

The 10MWT is a measure of mobility and walking speed. Starting in a standing position, 2 meters behind a timing device [Brower TC Timing System, Draper, Utah], participants walked as fast and as safely as possible for 14 meters. The time to complete the intermediate 10 meters was used to calculate walking speed (meters per second; m/s). Pre-, mid-, and post-testing 10MWT were performed with the same level of assistance.

\section{Walking independence}

The level of assistance used during the pre-test 10MWT was categorized using the valid and reliable WISCI II index. ${ }^{19-20}$ The WISCI II ranks walking independence from low independence (low score and high assistance) to independence (high score, no assistance). Before training following the mid-assessment and when returning study equipment after post-assessment, participants completed an untimed 10MWT with less assistance compared to the pre-test or mid-test, if possible. If less assistance was used, it was denoted on the WISCI II scale.

\section{Daily step physical activity}

Daily step physical activity was assessed on four consecutive days (three weekdays and one weekend day) with a Step Activity Monitor (SAM; Modus, Oklahoma City, Oklahoma). Data were not collected during any water activities or study training. The SAM is valid and reliable in those with iSCI. ${ }^{21}$ Participants affixed the SAM with a Velcro strap on their less-impaired leg after the investigator completed calibration procedures. ${ }^{22}$ The SAM recorded data in 1minute epochs. Data collection for DSPA started the day after receiving the SAM at pre-, mid-, and posttesting time points.

\section{Procedures}

To avoid muscular fatigue, participants were advised to avoid heavy physical activity 48 hours before the first visit. After assessing body mass, participants completed the informed consent, VAS, and did the TUG and
10MWT for familiarization. Participants were then introduced to the Eccentron and practiced the strength test protocol. Participants were given the SCIM to complete at home. Participants returned no sooner than 48 hours later for a second visit to repeat the TUG, 10MWT, and eccentric strength tests, this time for baseline data collection. At the end of this visit, participants were fitted with a SAM and briefed on care, application, and the schedule for wearing the device.

The ERT began with a two-week familiarization to avoid undue muscle soreness. Participants trained independently and face-to-face with the investigator. Participants started training at 50\% 1RM for two to three sets of eight repetitions at $12 \mathrm{rpm}$. Participants trained two times a week for 12 weeks. The ERT was designed to target both muscular strength and endurance as outlined by the National Strength and Conditioning Association. ${ }^{23}$ Exercise prescription was modified if the aforementioned training percentages were less than the participant's resting tone. In this instance, the same intensity change was added to the tone value and served as the new training workload (ex. tone $+35 \%$ and tone $+17 \%$ ). Intensity increased when the participant was able to accurately complete two additional repetitions in the last two sets on any given training day. However, if participants were unable to successfully generate the force needed or were inaccurate more than $30 \%$ of the session, intensity was reduced to the previously successful intensity. Participants trained and tested at approximately the same time of day each week.

\section{Statistical analyses}

An a priori power computation indicated that the analyses would be powered at $80 \%$ when 10 participants completed the ERT (G*Power, Version 3.1). Data were analysed with IBM SPSS Statistics for Windows (Version 23; Armonk, NY: IBM Corp). Descriptive statistics are provided for study variables in Table 3). Oneway repeated measures analysis of variances (ANOVAs) were used to determine the effect of ERT on the TUG, 10MWT, SCIM, and WISCI. Bivariate correlational analyses were conducted to determine the relationship between DSPA and the TUG, 10MWT, SCIM, and WISCI, respectively at pre-, mid, and posttest. Additional correlations were conducted to determine if the pre-test to post-test change in the TUG, 10MWT, SCIM, and WISCI scores, respectively, correlated with the change observed in DSPA (see Table 4). A familywise alpha of .05 determined statistical significance. Measures of effect were calculated $(d=$ difference 
Table 3 Means and standard deviations of outcome variables across the 12-week ERT.

\begin{tabular}{|c|c|c|c|c|c|c|}
\hline \multirow[b]{2}{*}{ Measure } & \multicolumn{2}{|c|}{ Pre-ERT } & \multicolumn{2}{|c|}{ Mid-ERT } & \multicolumn{2}{|c|}{ Post-ERT } \\
\hline & $\bar{M}$ & $S D$ & $\bar{M}$ & $S D$ & $\bar{M}$ & $S D$ \\
\hline TUG (secs) & 158.36 & 165.84 & 89.48 & 82.38 & 56.31 & 42.42 \\
\hline $10 \mathrm{MWT}(\mathrm{m} / \mathrm{s})$ & 0.34 & 0.42 & 0.39 & 0.44 & 0.43 & 0.50 \\
\hline WISCI & 8 & 7 & 10 & 7 & 13 & 7 \\
\hline SCIM & 22 & 10 & 23 & 10 & 24 & 10 \\
\hline Step activity & 1514 & 1899 & 1574 & 1879 & 1818 & 2163 \\
\hline
\end{tabular}

Note. ERT, eccentric resistance training; TUG, Timed Up and Go; 10 MWT, 10 meter walk test; WISCI, Walking Index for Spinal Cord Injury II; SCIM, Spinal Cord Independence Measure. Step activity $=$ steps taken per 1 limb.

Table 4 Pearson's correlation coefficients of DSPA to TUG, 10MWT, WISCI, and SCIM.

\begin{tabular}{llll}
\hline & \multicolumn{3}{c}{ Step activity } \\
\cline { 2 - 4 } Variable & Pre-test & Mid-test & Post-test \\
\hline Pre-TUG & -.608 & & \\
Pre-10MWT & .554 & & \\
Pre-WISCI & .609 & & \\
Pre-SCIM & .441 & $-.708^{*}$ & \\
Mid-TUG & & $.735^{*}$ & \\
Mid-10MWT & & $.829^{* *}$ & $-.689^{\star}$ \\
Mid-WISCI & & .494 & $.694^{*}$ \\
Mid-SCIM & & & -.613 \\
Post-TUG & & & .417 \\
Post-10MWT & & & \\
Post-WISCI & & & \\
Post-SCIM & & & \\
\hline
\end{tabular}

Note. Change represents difference from baseline to post-test. TUG, Timed Up and Go; 10MWT, 10 meter walk test; WISCI, Walking Index for Spinal Cord Injury; SCIM, Spinal Cord Independence Measure. Significance is denoted as * $=P<.05$ and $^{* *}=\mathrm{P}<.01$

in means/baseline standard deviation) to evaluate posttest values relative to pre-test values.

\section{Results}

Fourteen participants volunteered, of which 11 completed the ERT intervention. For personal reasons, three participants withdrew prior to completing the two-week familiarization period. Additionally, preDSPA data were lost for one participant as the result of a programming error. Therefore, analyses for DSPA had a sample size of 10 and analyses for ERT had a sample size of 11 . On average, the participants completed $98 \%$ of ERT sessions. There were no adverse events or elevated pain associated with the ERT.

The one-way repeated measures ANOVAs indicated significant ERT effects on TUG performance $(F(1.40$, $13.95)=4.93, \quad M S E=8664.94, \mathrm{H}-\mathrm{F} \mathrm{P}=.034)$ and 10MWT speed $(F(1.74,17.36)=7.92, M S E=0.004$, $\mathrm{H}-\mathrm{F} \mathrm{P}=.005)$. Post hoc analyses, corrected using the
Sidak procedure, failed to determine where the change occurred regarding TUG performance, but the pairwise comparisons found that change in 10MWT speed occurred from pre-test to post-test $(\mathrm{P}=.027)$. Participants also improved in WISCI scores from pretest to post-test $(F(1.80,18.00)=8.22, M S E=8.38$, $\mathrm{H}-\mathrm{F} \mathrm{P}=.004)$, but not in SCIM scores $(F(1.22$, $12.20)=0.87, M S E=33.14, \quad \mathrm{P}=.20)$. Additionally, the ERT did not have an effect on questions specific to mobility on the SCIM questionnaire, $(F$ (1.66, $16.56)=2.75, \mathrm{P}=.10)$. Correlational data are presented in Table 4. The improvement in 10MWT performance across the ERT was positively correlated with the change identified in DSPA $(r=.649, \mathrm{P}=.04$; see Figure 2).

\section{Discussion}

The ERT program was successful in improving performance on the TUG, 10MWT, and WISCI tests in those with chronic iSCI. Alternatively, the ERT had no effect on at home function. There were no relationships between study variables and DSPA before training. However, after implementing lower extremity ERT there was a significant relationship between DSPA and TUG, 10MWT, and/or WISCI values, respectively, at mid-test and post-test. Further, the improvement in 10MWT walking speed was positively correlated with the improvement in DSPA.

Improvement in TUG performance from pre-test to post-test $(\sim 45 \%)$ appeared to occur linearly from pretest to mid-test $(\sim 25 \%)$ and from mid-test to post-test $(\sim 23 \%)$. Meaningful change on the TUG by individuals with iSCI was determined to be a minimum of 10.8 seconds or $30 \%{ }^{24}$ Other RT programs have elicited improvements in TUG performance in populations with chronic pain and neurological dysfunction, similar to iSCI. A sample of participants with rheumatoid arthritis completed a 14 session, concentric only RT program targeting the hamstrings and the quadriceps. ${ }^{25}$ These participants improved on the TUG by $12.5 \%$ with a concurrent $80 \%$ drop in pain (measured via VAS). Although not statistically analysed, individuals in the current sample also reported lower levels of daily pain/discomfort (as measured by VAS) over the course of the ERT. Future investigations may be designed to specifically elucidate this potential connection between RT and reduction in pain, specifically, looking into neuropathic pain experienced after iSCI.

The continual improvement in TUG performance may have resulted from the development of lower extremity strength and explosiveness. ${ }^{25}$ The ability to propel out of a seated position and subsequently perform gait 


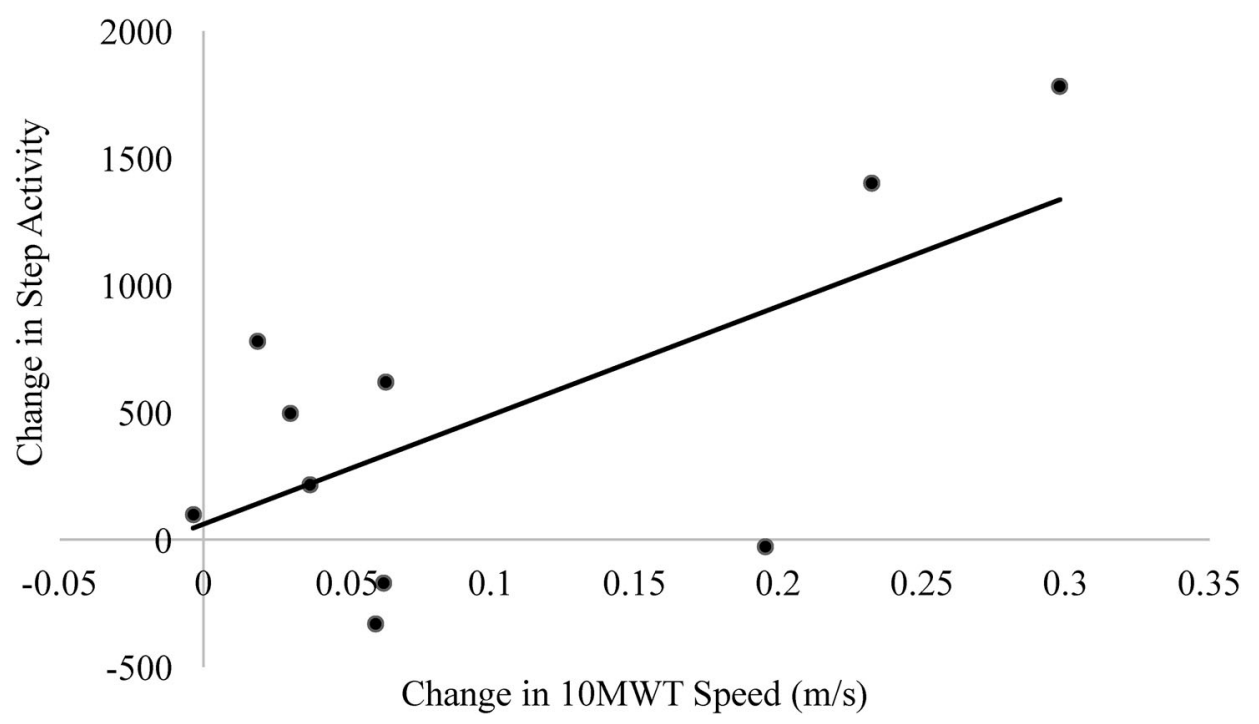

Figure 2 Change scores are different in performance from baseline to post testing. Step activity represents steps taken across 3 week days and 1 weekend day by less impaired limb. 10MWT $=10$ meter walk test. Correlation of $r=.649$ significant at $P=.04$.

mechanics may be derivatives of neural and architectural adaptations to the local musculature. McHugh and Tetro $^{26}$ noted the addition of sarcomeres longitudinally to myofibrils is as little as 10 days following ERT while others quantified increased agonist activation and attenuated antagonistic coactivation after 4 weeks of ERT. ${ }^{27-28}$ As these variables were not assessed in this investigation, further research should evaluate both neuroplasticity and local musculoskeletal adaptations to understand the efficacy of ERT in samples that include individuals with iSCI.

Walking speed on the 10MWT improved from $0.34 \mathrm{~m} / \mathrm{s}$ at pre-test to $0.39 \mathrm{~m} / \mathrm{s}$ at mid-test, to $0.43 \mathrm{~m} / \mathrm{s}$ at post-test, resulting in a significant overall improvement $(\sim 26.5 \%$, respectively). A minimum change of $0.13-0.17 \mathrm{~m} / \mathrm{s}$ or $39 \%$ is needed to be considered meaningful for those with iSCI. ${ }^{24,29}$ It is important to note that these standards were developed in individuals post-iSCI with faster initial walking speeds (average speed $=0.81 \pm 0.34 \mathrm{~m} / \mathrm{s}$ ). ${ }^{30}$ Despite falling short of the predetermined benchmark for meaningful change in ambulatory speed, individuals in the current sample improved walking speed following a 12-week ERT, without formal gait training.

Stevens et al $^{22}$ noted that freely chosen walking speed during a 10MWT was associated with DSPA in those with longstanding cervical, thoracic, or lumbar iSCI. They identified $0.42 \mathrm{~m} / \mathrm{s}$ as a walking speed threshold for the start of a curvilinear increase in DSPA with minor improvements in gait speed. The current ERT improved walking speed, bringing the sample average to $0.43 \mathrm{~m} / \mathrm{s}(0.01-1.3 \mathrm{~m} / \mathrm{s})$. While speculative, a longer duration of ERT may bring about faster walking and higher volumes of DSPA. Overall, the lower therapist and patient time investment accompanied by similar gait improvements following ERT supports implementation in the therapeutic realm.

After engaging in the ERT, scores on the TUG, 10MWT, and WISCI were highly correlated with DSPA midway through training with correlations remaining at post-test for TUG and 10MWT scores. The lack of pre-test correlations may be due to the variability in participants' daily steps (1,514 $\pm 1,898$ steps $)$ and general lower extremity weakness (summed eccentric strength averaged $152.8 \pm 122.2 \mathrm{lbs}$ and summed isometric strength averaged $8.2 \pm 7.7 \mathrm{~N} / \mathrm{kg}$ ). The lack of lower extremity strength at baseline might have brought about a floor effect on DSPA. However, as participants gained strength from the ERT (compared to pre-test, mid-eccentric strength improved $22.7 \%$ and post-test improved $37.8 \%$ ), they responded similarly, thus increasing the ability to detect correlations.

This novel approach of using laboratory measures (WISCI, TUG, and 10MWT) to identify relationships with DSPA differs from previous literature. Investigations have previously determined relationships between measurement tools themselves, i.e. 10MWT to TUG or TUG to WISCI. This alternate approach may allow clinicians to predict DSPA from laboratorybased gait assessments. Future investigations are needed to determine the direction of the relationship and capacity of the TUG, 10MWT, and WISCI to predict DSPA as lower extremity strength improves in those with iSCI. 
On average, individuals in the current sample substantially improved on the WISCI scale after the 12-week ERT ( $\mathrm{M}=5 \pm 5$ points; $d=.73$ ). A change of 1 point is a meaningful difference in ambulatory individuals with acute and chronic iSCI. ${ }^{31}$ In comparison, robotic assisted BWSTT for 4 weeks improved WISCI scores an average of 7 points in three individuals with varying severity of chronic iSCI. ${ }^{32}$ Both interventions resulted in improvements in ambulatory independence. Due to the variability within this population relative to baseline walking ability, walking speed, and duration and severity of injury, continued investigation is needed to evaluate how training and/ or therapy can improve gait independence.

In the current sample, there was a non-significant $4.5 \%$ average improvement on the SCIM. While a meaningful change has yet to be defined on the SCIM, 12 individuals with iSCI (complete tetraplegia to thoracic) subjectively identified their personal, meaningful change on the scale. ${ }^{33}$ Their meaningful changes ranged from 1 to 7 points (approximately $2.5-18.2 \%$ ) on the mobility subscale. These data may serve as initial benchmarks for clinically meaningful differences on the SCIM. ${ }^{33}$

\section{Conclusions}

The 12 weeks of ERT served as a sufficient stimulus to improve measures of walking speed and independence without formal gait training, in those with iSCI. Individuals with chronic iSCI may expect to improve mobility by an average of 45\% (TUG), walking speed by $39 \%$ (10MWT), and walking independence by $134 \%$ (WISCI) if a similar ERT is completed. Current participants improved at home function by an insignificant average of 4\% (SCIM). No correlations existed between DSPA and 10MWT, TUG, WISCI, or SCIM measures prior to lower extremity exercise training. However, correlations were significant after the implementation of ERT. These data support the implementation of ERT, as an adjunct to formal gait training, in therapeutic regimens seeking to improve walking capacity in those with iSCI with minimal therapist burden. Future endeavours, with sufficient control conditions, to assess neuroplasticity and musculoskeletal adaptations to ERT and other modalities of training (concentric, NMES, robotic therapy, etc.) are encouraged.

\section{Disclaimer statements}

Funding No funding agencies were involved in the process of this investigation.

Conflicts of interest The authors have conflicts of interest to disclose.

\section{References}

1 Field-Fote EC. Spinal cord injury rehabilitation. Philadelphia: F.A. Davis Company. 2009.

2 Saunders LL, Clarke A, Tate D, Forchheimer M, Krause JS. Lifetime prevelance of chronic health conditions among persons with spinal cord injury. Arch Phys Med Rehabil 2015; 96:673-679.

3 Jacobs P, Nash M. Exercise recommendations for individuals with spinal cord injury. Sports Med. 2004;34:727-51.

4 Ginis KAM, Hicks AL, Latimer AE, Warburton DER, Bourne C, Ditor DS, et al. The development of evidence-informed physical activity guidelines for adults with spinal cord injury. Spinal Cord 2011;49:1088-96.

5 American College of Sports Medicine. ACSM's guidelines for exercise testing and prescription. Philadelphia: Wolters Kluewer. 2017.

6 Gorgey AS, Mather KJ, Cupp HR, Gater DR. Effects of resistance training on adiposity and metabolism after spinal cord injury. Med Sci Sports Exerc 2012;44(1):165-174.

7 Gorgey AS, Caudil C, Khalil RE. Effects of once weekly NMES training on knee extensors fatigue and body composition in a person with spinal cord injury. J Spinal Cord Med. 2016;39(1): 99-102.

8 Dudley GA, Castro MJ, Rogers S, Apple DF. A simple means of increasing muscle size after spinal cord injury: A pilot study. Eur J Appl Physiol 1999;80:394-396.

9 Gorgey AS, Shepard C. Skeletal muscle hypertrophy and decreased intramuscular fat after unilateral resistance training in spinal cord injury: Case report. J Spinal Cord Med 2010;33:90-95.

10 Labruyere R, van Hedel HJ. Strength training versus robot-assisted gait training after incomplete spinal cord injury: A randomized pilot study in patients depending on walking assistance. J Neuroeng Rehab 2014;11(4):1-12.

11 Enoka RM. Eccentric contractions require unique activation strategies by the nervous system. J App Physiol 1996;81:2339-46.

12 Lepley LK, Palmieri-Smith RM. Effects of eccentric strengthening after Anterior Cruciate Ligament reconstruction on quadriceps strength. J Sport Rehabil 2013;22:150-156.

13 Stone WJ, Stevens SL, Fuller DK, Caputo JC. Eccentric resistance training in adults with and without spinal cord injuries. Int J Exerc Sci 2017;10(1):154-165.

14 Bryce TN, Budh CN, Cardenas DD, Dijkers M, Felix ER, Finnerup NB. Pain after spinal cord injury: An evidence-based review for clinical practice and research. J Spinal Cord Med 2007;30(5):421-440.

15 Catz A, Itzkovich M, Steinberg F, Philo O, Ring H, Ronen J et al. The Catz-Itzkovich SCIM: A revised version of the Spinal Cord Independence Measure. Disabil Rehabil 2001;23(6):263-268.

16 Wirz M, Muller R, Bastiaenen CHG. Falls in persons with spinal cord injury: Validity and relability of the Berg Balance Scale. Neurorehab Neural Rep 2010;24(1):70-77.

17 Brzycki M. Strength testing- predicting a one-rep max from repsto-fatigue. J Phys Health Educ Recreat Dance 1993;64:88-90.

18 van Hedel HJ, Wirz M, Dietz V. Assessing walking ability in subjects with spinal cord injury: Validity and reliability of 3 walking tests. Arch Phys Med Rehabil 2005;86(2):190-196.

19 Ditunno JF, Ditunno PL, Graziani V, Scivoletto G, Bernardi M, Catellano V, et al. Walking index for spinal cord injury (WISCI): an international mutlicenter validity and reliability study. Spinal Cord 2000;38(4):234-243.

20 Ditunno PL, Dittuno JF. Walking index for spinal cord injury (WISCI II): scal revision. Spinal Cord 2001;39:654-656.

21 Bowden MG, Behrman AL. Step Activity Monitor: Accuracy and test-retest reliability in persons with incomplete spinal cord injury. J Rehabil Res Dev 2007;44:355-62.

22 Stevens SL, Caputo JL, Fuller DK, Morgan DW. Effects of underwater treadmill training on leg strength, balance, and walking performance in adults with incomplete spinal cord injury. J Spinal Cord Med 2015;38:91-101.

23 Haff GG, Triplett NT. Essentials of strength training and conditioning $\left(4^{\text {th }}\right.$ ed.). Champaign: Human Kinetics. 2016.

24 Lam T, Noonan VK, Eng JJ, the SCIRE Research Team. A systematic review of functional ambulation outcome measures in spinal cord injury. Spinal Cord 2008;46:246-54. 
25 McMeeken J, Stillman B, Story I, Kent P, Smith J. The effects of knee extensor and flexor muscle training on the timed-up-and-go test in individuals with rheumatoid arthritis. Physiother Res Int 1999;4:55-67.

26 McHugh P, Tetro D. Changes in the relationship between joint angle and torque production associated with the repeated bout effect. J Sports Sci 2003;21(11):927-932.

27 Krentz JR, Farthing JP. Neural and morphological changes in response to a 20-day intense eccentric training protocol. Eur $\mathbf{J}$ App Physiol 2010;110:333-340.

28 Pensini M, Martin A, Maffiuletti NA. Central versus peripheral adaptations following eccentric resistance training. Int $\mathbf{J}$ Sports Med 2001;23:567-574.

29 Musselman KE, Yang, JF. Spinal cord injury functional ambulation profile: A preliminary look at responsiveness. Physical Therapy 2014;94:240-50.
30 Lemay JF, Nadeau S. Standing balance assessment in ASIA D paraplegic and tetraplegic participants: Concurrent validity of the Berg Balance Scale. Spinal Cord 2010;48:245-50.

31 Burns AS, Delparte JJ, Patrick M, Marino RJ, Ditunno JF. The reproducibility and convergent validity of the walking index for spinal cord injury (WISCI) in chronic spinal cord injury. Neurorehab Neural Repair 2011;25:149-57.

32 Hornby TG, Zemon DH, Campbell D. Robot-assisted, body-weight-supported treadmill training in individuals following motor incomplete spinal cord injury. Phys Ther 2005; 85:52-66.

33 Corallo V, Torre M, Ferrara G, Guerra F, Nicosia G, Romanelli E, et al. What do spinal cord injury patients think of their improvement? A distribution and anchor based study of the minimally clinically important difference of the Spinal Cord Independence Measure III (SCIM III). Eur J Phys Rehabil Med in press 2017. 